\begin{tabular}{|c|c|}
\hline Title & Estimation of Gillnet Selectivity Curve by Maximum \\
\hline Author(s) & Fujimori, Y asuzumi; Tokai, Tadashi \\
\hline Citation & $\begin{array}{l}\text { Fisheries Science, 67(4), 644-654 } \\
\text { https://doi.org/10.1046/.1444 2906.2001.00301.x }\end{array}$ \\
\hline Issue Date & $2001-08$ \\
\hline Doc URL & http:/hdl.handle.net/2115/622 \\
\hline Rights & Copyright (c) 2001 the Japanese Society of Fisheries Science（日本水産学会） \\
\hline Type & article (author version) \\
\hline Additional Information & There are other files related to this item in HUSCAP. Check the above URL. \\
\hline File Information & Manuscript.pdf (本文) \\
\hline
\end{tabular}

Instructions for use 


\title{
Estimation of Gillnet Selectivity Curve by Maximum Likelihood Method
}

\author{
Yasuzumi Fujimori, ${ }^{*}$ Tadashi Tokai $^{2}$
}

Running Title: Gillnet Selectivity Estimation

${ }^{1}$ Division of Marine Environment and Resources, Hokkaido University, Hakodate, Hokkaido 041-8611, Japan

${ }^{2}$ Department of Marine Science and Technology, Tokyo University of Fisheries, Minato, Tokyo 108-8477, Japan

*Corresponding author: Tel: 0138-40-8832. Fax: 0138-40-8832. Email:

fujimori@fish.hokudai.ac.jp 


\begin{abstract}
A maximum likelihood method of estimating gillnet selectivity when the data are obtained by gillnet fleets consisting of several nets of differing mesh size is presented in this paper. The SELECT model is expanded by application of the relative length (i.e. the ratio of fish length to mesh size) to obtain a master curve of gillnet selectivity. Four kinds of functional model, normal, lognormal, skew-normal and bi-normal are fitted to the data. In addition, two cases where the relative fishing intensity is either estimated or fixed by catch effort are compared. The bi-normal model has the lower model deviance regardless of whether the relative fishing intensity is estimated or not. The estimation of the relative fishing intensity by catch effort is also examined where the estimates of the parameter of the SELECT model are compared with the catch effort as determined by the number of nets of each mesh size used. For the bi-normal model these quantities compare well. Thus, it is concluded that this method gives reliable estimates even if the data for each mesh size is obtained with different catch efforts.
\end{abstract}

\title{
Keywords: bi-normal, catch effort, gillnets, maximum likelihood method, selectivity curve, SELECT model
}




\section{INTRODUCTION}

Many methods of estimating gillnet selectivity have been reported. These have been classified by Hamley ${ }^{1}$ into five categories. Under this classification, the method of comparing the fish size compositions captured by two or more mesh sizes is included with the indirect methods. Many researchers use this method because estimates of selectivity can be obtained from the catch data without having to make strict assumptions on the fish population distribution or having to carry out complex calculations. Some development of indirect method is continued still. ${ }^{2,3}$ Almost all the method is based on the geometrical similarity theory of Baranov ${ }^{4}$ which assumes that selection is a function of the ratio of fish body size and mesh size. Holt ${ }^{5}$ developed an early estimating method based on this theory. A similar method was proposed by Ishida ${ }^{6}$ and Kitahara ${ }^{7}$ in Japan during the same period. These methods are still often used because of ease of calculation. ${ }^{8-12}$

The methods of Ishida ${ }^{6}$ and Kitahara ${ }^{7}$ assume that the fishing intensity of each mesh size in a fleet of gillnets consisting of different mesh sizes are equal. This means that there is an equal chance of fish encounter at any mesh size and also that the gear efficiency of each mesh size is equal. Fujimori et al. ${ }^{12}$ expand the method of Kitahara ${ }^{7}$ to take into account the difference of gear efficiency of each mesh size. Hence, these methods can only be applied to data sets where the catch effort at each mesh size is the same or to data sets that have been standardized by catch effort, at each mesh size. In such experiments, the gillnets are fished two or more times 
for a certain length of time and usually the aggregated data is used to analyze the gillnet selection. The numbers of nets at each mesh size, however, are not necessarily equal. Ishida ${ }^{6}$ and Kitahara ${ }^{7}$ therefore used CPUE to standardize the data in their analyses where the number of nets is assumed to be a measure of catch effort.

The catching of gillnets is also influenced by the interaction of fish behavior and environmental conditions such as current, illuminance, etc. ${ }^{13,}$ 14 This suggests that the likelihood of fish encounter to a net during each fishing operation will not be constant, and, therefore, that the fishing intensity will vary at each operation. In addition, there may be competition for fish between nets with different mesh sizes. ${ }^{15}$ Pope et al. ${ }^{16}$ proposed operational methods to decrease this competition, and Kitahara ${ }^{7}$ examined the bias due to net position of one mesh size in a fleet and corrected the catch data based on this result. In addition, it is known as a matter of course that gear efficiency changes as mesh size increases. ${ }^{10,15-17}$ These cases show that it is unrealistic to assume that the fishing intensity is constant at any mesh size. Hence, standardizing the numbers of fish caught with a catch effort measure based on the number of nets used or some other fixed condition such as number of meshes or net length is likely to over or underestimate the catch of some mesh sizes.

Wulff ${ }^{18}$ assumed that gillnet capture increased in accordance with a Poisson distribution, and estimated the selectivity parameters and their variances by the maximum likelihood method. We cannot use CPUE in the maximum likelihood calculation because it is not an actual observed 
quantity. The data used for estimating the selectivity parameters should only be the observed catch data obtained under conditions of equal catch effort at each mesh size, otherwise the variation of catch effort at each mesh size would have to be considered. Millar ${ }^{19-21}$ employed an individual parameter to represent the fishing intensity of different gears used simultaneously (SELECT model). This parameter includes the influences of catch effort and gear efficiency. Millar and Fryer $^{22}$ have also demonstrated the application of SELECT model to gillnet data where the relative fishing intensity is defined as a function of gillnet mesh size.

Nishiuchi ${ }^{23}$ estimated the selectivity curve of hair crab traps by application of the SELECT model to the master curve method. ${ }^{24}$ He dealt with the parameter of relative fishing intensity of each mesh size individually and showed an improvement of the accuracy of the parameter estimates. Generally, the selectivity curve of crab traps is described by a logistic function (the same as for trawl nets), which can be estimated by comparative fishing experiments using two different gear specifications. ${ }^{22}$, 23 For gillnets, three mesh sizes or more are necessary to obtain satisfactory results. This is due to the fact that the selection range of gillnets is wider than those of the gears mentioned above. Hence it is thought that using a SELECT model in conjunction with a multinomial distribution $^{22}$ would better estimate gillnet selectivity. It is necessary, however, to determine the choice of function with which to represent the gillnet selectivity curve.

This paper describes a method of estimating a master curve of gillnet 
selectivity from the catch data obtained by a fleet of gillnets consisting of different mesh sizes by application of the fish length / mesh size ratio ${ }^{23}$ to the SELECT model. Four kinds of functional models are applied to the selectivity curve and the optimal model is examined by evaluating the model deviance. The catch data from six kinds of mesh size, each with a different catch effort and a different number of nets used, are analyzed.

\section{ESTIMATING METHOD}

\section{General model}

Let $c_{i j}$ be the number of fish of length $l_{j}$ caught in a net with mesh size $m_{i}$. Hence

$$
c_{i j}=q_{i} x_{i} \lambda_{j} S_{i j}
$$

where $\lambda_{j}$ is the number of fish of length $l_{j}$ that come into contact with the net, $S_{i j}$ is the selectivity of the net with mesh size $m_{i}$ to fish of length $l_{j}, q_{i}$ is the gear efficiency and $x_{i}$ is the relative catch effort. Defining $p_{i}$, the relative fishing intensity to be $p_{i}=q_{i} x_{i}$ gives

$$
c_{i j}=p_{i} \lambda_{j} S_{i j}
$$

where $\sum_{i=1}^{k} p_{i}=1 . k$ is the number of mesh sizes used. Assuming the principle of geometric similarity permits the selectivity curve to be expressed as follows

$$
\begin{aligned}
S_{i j}= & S\left(m_{i}, l_{j}\right) \\
& =s\left(R_{i j}\right)
\end{aligned}
$$

where $R_{i j}=l_{j} / m_{i}$ and $s\left(R_{i j}\right)$ is in the range $0<s\left(R_{i j}\right) \leq 1$. Kitahara ${ }^{7}$ called the 
selectivity curve of this type a master curve, which is based on length to mesh size ratio (relative length). Substituting eq.(3) into eq.(2) produces

$$
c_{i j}=p_{i} \lambda_{j} s\left(R_{i j}\right)
$$

\section{Selectivity curve model}

Generally, the selectivity curve of gillnets has been assumed to be a unimodal curve. A number of functional forms have been employed which are either symmetric such as the normal function ${ }^{5,22}$ or asymmetric such as lognormal function, ${ }^{21,26}$ skew-normal function, ${ }^{2,3,18,25}$ and gamma function. ${ }^{22,25}$ Lately, the application of bimodal curve has also been reported. ${ }^{10,22,27}$

In this study, four kinds of function are used as a selectivity curve model. These are divided into two types of function, unimodal function (the normal, the lognormal, and the skew-normal function) and bimodal function (the bi-normal function).

The equation of the normal, lognormal, and skew-normal model are shown below.

$$
\begin{gathered}
s\left(R_{i j}\right)=\exp \left(-\frac{\left(R_{i j}-R_{0}\right)^{2}}{2 \sigma^{2}}\right) \\
s\left(R_{i j}\right)=\exp \left(-\frac{\left(\ln R_{i j}-\ln R_{0}\right)^{2}}{2 \sigma^{2}}\right) \\
s\left(R_{i j}\right)=\exp \left(-\frac{\left(R_{i j}-R_{0}\right)^{2}}{2 \sigma^{2}}\right) \cdot\left\{1-1 / 2 \eta \sigma^{3 / 2} \cdot\left(\frac{\left(R_{i j}-R_{0}\right)}{\sigma}-\frac{\left(R_{i j}-R_{0}\right)^{3}}{3 \sigma^{3}}\right)\right\}
\end{gathered}
$$

where, $R_{0}$ in each model is the relative length with the maximum value 
$(=1.0)$ of the selectivity curve and $\sigma$ is the parameter that decides the curve width. The parameter $\eta$ in the skew-normal model is the skewness constant.

The equation of the bi-normal model is given by

$$
s\left(R_{i j}\right)=\frac{1}{\delta}\left[\exp \left(-\frac{\left(R_{i j}-R_{a}\right)^{2}}{2 \sigma_{a}^{2}}\right)+\omega \exp \left(-\frac{\left(R_{i j}-R_{b}\right)^{2}}{2 \sigma_{b}^{2}}\right)\right]
$$

Here, $R_{a}$ and $R_{b}$ are the relative length with the maximum value of each constituent normal curve and $\sigma_{a}$ and $\sigma_{b}$ are the parameters that decide the width of both these curves respectively. The weighting factor $\omega$ decide the height of second curve, and $\delta$ the scaling constant to make the maximum value of the selectivity 1.0 .

\section{Fitting the model by maximum likelihood method}

Let $C_{j}$ be the total number of fish of length $l_{j}$ that are caught by a gillnet fleet that consists of panels of several mesh sizes. Hence:

$$
C_{j}=\sum_{i=1}^{k} c_{i j}
$$

The probability that a fish of length $l_{j}$ is caught by each mesh size as $c_{1 j}$, पर०, $c_{k j}$ is described by the following multinomial distribution.

$$
P_{j}=\frac{C_{j} !}{\prod_{i=1}^{k} c_{i j} !} \prod_{i=1}^{k} \phi_{i j}{ }^{{ }^{i j}}
$$

Here, $\phi_{i j}$ is the proportion of fish of length $l_{j}$ captured in a mesh of size $m_{i}$ and has the equation:

$$
\phi_{i j}=c_{i j} / C_{j}=c_{i j} / \sum_{i=1}^{k} c_{i j}
$$


When Eq.(10) is applied to each length $l_{j}$ individually, the total probability is given by

$$
P=\prod_{j=1}^{n} P_{j}=\prod_{j=1}^{n}\left(\frac{C_{j} !}{\prod_{i=1}^{k} c_{i j} !} \prod_{i=1}^{k} \phi_{i j}{ }^{{ }^{i j}}\right)
$$

where, $n$ is the number of length classes. Substituting the general model Eq.(4) into Eq.(11), the estimated proportion of fish caught with relative length can be obtained as:

$$
\begin{aligned}
\phi\left(R_{i j}\right) & =p_{i} \lambda_{j} s\left(R_{i j}\right) / \sum_{i=1}^{k} p_{i} \lambda_{j} s\left(R_{i j}\right) \\
& =p_{i} s\left(R_{i j}\right) / \sum_{i=1}^{k} p_{i} s\left(R_{i j}\right)
\end{aligned}
$$

Then, the parameters of selectivity model and relative fishing intensity $p_{i}$ are estimated by maximizing the following

$$
L=\prod_{j=1}^{n}\left(\frac{C_{j} !}{\prod_{i=1}^{k} c_{i j} !} \prod_{i=1}^{k}\left(p_{i} s\left(R_{i j}\right) / \sum_{i=1}^{k} p_{i} s\left(R_{i j}\right)\right)\right.
$$

For practical calculation it is the following log-likelihood, without the constant term, that is maximized

$$
\log _{e} L=\sum_{j=1}^{n} \sum_{i=1}^{k}\left(c_{i j} \log _{e}\left(p_{i} s\left(R_{i j}\right) / \sum_{i=1}^{k} p_{i} s\left(R_{i j}\right)\right)\right)
$$

Two models: one where the values of $p_{i}$ are regarded as estimates; and the other where the values of $p_{i}$ are fixed by use of the relative catch effort are compared in this study. When $p_{i}$ is assumed fixed, the relative catch effort is obtained from the number of nets used in every mesh size, $o_{i}$, that is, $x_{i}=o_{i} / \sum_{i=1}^{k} o_{i}$. 


\section{Model deviance}

Model deviance is evaluated from the residual differences between $\phi_{i j}$ calculated directly from the data and $\phi\left(R_{i j}\right)$ from the model. Hence, the deviance residual is given as follows. ${ }^{28}$

$$
d_{i j}= \pm\left[2 C_{j}\left(\phi_{i j} \log _{e}\left(\frac{\phi_{i j}}{\phi\left(R_{i j}\right)}\right)+\left(1-\phi_{i j}\right) \log _{e}\left(\frac{1-\phi_{i j}}{1-\phi\left(R_{i j}\right)}\right)\right)\right]^{1 / 2}
$$

The sign of the right side is depending on whether $\phi_{i j}$ is greater (plus) or less than $\phi\left(R_{i j}\right)$ (minus).

The deviance residuals obtained from Eq.(16) are represented for each mesh size and length respectively by which the values of $R_{i j}$ are multiplied by mesh size $m_{i}$. If the deviance residuals $d_{i j}$ are clustered on one-side with positive or negative area, the model is judged unsuitable. ${ }^{29,30}$ A goodness of fit for each model was assessed using the plot of deviance residuals and the model deviance as follows:

$$
D=\sum_{i=1}^{k} \sum_{j=1}^{n}\left(d_{i j}\right)^{2}
$$

Additionally, the model deviance is referred to a chi-square distribution with the degree of freedom (d.f.). The value of d.f. is calculated from $n \times(k-1)-r, r$ is the number of model parameters, when $p_{i}$ is fixed and $n \times(k-1)-(r+k-1)$ when $p_{i}$ is estimated. Here, $k$ is the number of $p_{i}$, i.e. the number of different mesh sizes used and $n$ is the number of length classes. The estimation of parameter using Eq.(15) is conducted by the Solver add-on program in MS-Excel. 


\section{Catch data}

Table 1 gives the catch of pink salmon Oncorhynchus gorbushia from a stock investigation by Oshoro-Maru of Hokkaido University in 1957. These data were obtained from six types of gillnet of differing nominal mesh size $(7.6,9.6,10.6,12.1,12.7$ and $13.6 \mathrm{~cm})$ and were used by Ishida ${ }^{6}$ to estimate selectivity. Ishida uses only the data in the fork length range of 41.5 to $47.5 \mathrm{~cm}$ although the original data set comprises fish in the range 36.5 to $56.5 \mathrm{~cm}$. Ishida transformed this data to CPUE (catch per one hundred net) in his calculation since the number of nets used for each mesh size was different (see Table 1). However, we do not standardize the data because the method we propose is able to estimate the difference of fishing intensity in each mesh size.

\section{RESULTS}

The estimated parameters of each function model are shown in Table 2. In each case the model deviance were smaller when the $p_{i}$ were estimated than when they were fixed (the parameters of the skew-normal model with the $p_{i}$ fixed was not converged). It is thought that, the estimates of the model parameters are more reliable when the $p_{i}$ are estimated. Comparing each function model, the bi-normal model showed the lowest model deviance regardless of whether the $p_{i}$ was estimated or not, although its model deviance is higher than its degree of freedom as same as that of other model $(P<0.01$, chi-square test $)$.

Fig. 1 shows the master curve of selectivity for each model when the $p_{i}$ 
was fixed. The bi-normal model is similar in modal relative length to the normal and skew-normal model, although, as would be expected, its overall shape differs. The modal relative length of the lognormal model is larger than that of the other models. The observed and estimated probability of capture, $\phi_{i j}$ and $\phi\left(R_{i j}\right)$, are compared in Fig. 2(a) and (b). The results of the unimodal models are all fairly similar. The lognormal model provides a better fit than either the normal or skew-normal for the $7.6 \mathrm{~cm}$ mesh size data, and has smaller model deviance. Overall, however, the bi-normal model is the best fit and gives a large reduction in model deviance compared with the unimodal models. It is inferred naturally that the modal length of these probabilities will be large as the mesh size expands. Such a tendency is seen clearly in the bi-normal model although the probabilities of the $13.6 \mathrm{~cm}$ mesh size are slightly larger than that of $12.7 \mathrm{~cm}$ (Fig. 2). Fig. 3 plots the values of the deviance residuals, $d_{i j}$, in each model calculated from Eq.(16). The residuals of the unimodal models are very similar with a bias towards positive values on the right hand side and negative values on the left hand side of the plots. On the other hand, the residuals of the bi-normal model do not demonstrate any such tendency and there is a corresponding improvement in the model deviance (Table 2).

The relative fishing intensity, $p_{i}$, is assumed to reflect the influence of catch effort and gear efficiency. However, in this study, it is thought that $p_{i}$ mainly reflects the effect of catch effort alone. This is because the number of nets used is a decisive factor affecting the catch size. Accordingly, the $p_{i}$ estimates are compared with the relative catch effort 
(see Table 1). On making this comparison, in effect, we are assuming that $q_{i}=1$ and that $p_{i}=x_{i}$. The $p_{i}$ estimates of the normal and skew-normal model are very poor fits to the catch effort values (Fig. 4). This is particularly the case for the $p_{1}$ value. There is a slight improvement with the lognormal model, however, the best agreement is found with the bi-normal model.

\section{DISCUSSION}

\section{Validity of the estimating model}

In any model, the model deviance is substantially higher than its degree of freedom $(P<0.01$, chi-square test $)$ and the residuals shown in Fig. 3 does suggest some lack of fit even in the bi-normal model. It will be one of the reasons that the data of each mesh size in this study was not taken with the same experimental condition; the combination of mesh size was varied between hauls. However, it is clear that estimating the relative fishing intensity, $p_{i}$, improves the fit of the selectivity curve to the data. Furthermore, the bi-normal model is most appropriate to the pink salmon data used in this study. In Fig. 5 the selectivity curves for the $12.1 \mathrm{~cm}$ mesh, estimated from the master curve of each model, are compared with the selectivity plots calculated from Ishida's method. ${ }^{6}$ Ishida's results have not been adjusted by the coefficient of population density. ${ }^{31}$ They are very helpful in understanding the characteristics of the data as they are obtained from the catch probabilities between nets without any correction and without any assumptions concerning the curve shape. The modal length of each selectivity curves examined in this study, except for the lognormal 
model, corresponds well with the plot obtained from Ishida's method. However, only the bi-normal model identifies the high relative efficiency at the larger lengths. The bi-normal model also has the lowest deviance and its estimates of relative fishing intensity compare best with the values of the relative catch effort. Therefore, it is concluded that the bi-normal model where the relative fishing intensity, $p_{i}$, is estimated is appropriate to the data used in this study.

This method is capable of estimating fishing effort when the optimal function model is used to estimate the selectivity curve. The relative fishing intensity, however, is not equivalent to the relative catch effort because it is not certain that the gear efficiency, $q_{i}=1$. Millar and Fryer ${ }^{22}$ used the data obtained from six kinds of mesh size with the same number of nets. They supposed that the relative fishing intensity was a function of mesh size because due to the fact that there was the same number of meshes in each net, the lengths of their nets were proportional to the mesh size. However, they did not show an improvement of estimating accuracy.

The relative fishing intensity in this study includes a contribution from both gear efficiency and relative catch effort, i.e. $p_{i}=q_{i} x_{i}$. Therefore, if the contribution of the catch effort is smaller than that of the gear efficiency, the relative fishing intensity will not accurately characterize the effort factor. As in this study, the influence of catch effort might have to be analyzed after the relative fishing intensity of each mesh size is obtained individually and before the number of parameter is decreased by the assumption that the relative fishing intensity will be a function of mesh 
size.

\section{Shape of selectivity curve}

Almost all the selectivity curve of gillnets in recent studies are represented by an asymmetrical curve model. 2,3,17,18 However, in this study the lognormal model and the skew-normal model did not suit the data owing to the extreme skewness of data. The bi-normal model is the best for this data. The bimodal curve is usually used to model the selectivity of gillnets where fish entanglement plays a large part. ${ }^{10,27}$ Generally, the mesh does not select the same body part of fish and accordingly if the girth at these parts is greatly different, the skewness of curve will be large. Yamamoto and Mishima ${ }^{32}$ were able to distinguish, from wounds on the body of pink salmon, where the gillnet caught the fish: either between the operculum and the pectoral fin; or between the pectoral fin and the dorsal fin. Moreover, they have shown that the catch frequency of each region was not equal and changed with the seasons. If it is assumed that a girth of 24.2 $\mathrm{cm}$ is the optimum girth that is caught in a mesh size of $12.1 \mathrm{~cm}$, the effect of fish being caught at different parts of the body can be examined. The fork length of a pink salmon with a $24.2 \mathrm{~cm}$ operculum girth is $48.7 \mathrm{~cm}$ whereas that for a fish with a $24.2 \mathrm{~cm}$ body girth is $42.2 \mathrm{~cm} .^{32}$ The difference of fork lengths is $6.5 \mathrm{~cm}$, which is close to the difference between the two modal lengths $(=8.5 \mathrm{~cm})$ of the bi-normal model (Fig. 5). Thus, it is thought that such multiple part selection causes bi-normal selectivity in gillnets. Such a capture process of gillnet has reported by Hovgård. ${ }^{33}$ 
Further investigation is needed to clarify the details of gillnet capture process to decide a selectivity curve precisely.

This method does not need any standardization of catch data in calculation of the selectivity, even if the catch effort of each mesh size is different. Moreover, the difference of catch effort can be estimated by the relative fishing intensity, and if the data obtained by the same catch effort is used, the bias of gear efficiency in each mesh size may be evaluated, although a large data might be necessary for calculation to obtain a statistical reliability. The use of an appropriate model for selectivity curve is a prerequisite to evaluate the catch effort and the gear efficiency accurately. Accordingly, it is necessary to use a range of function models for the selectivity curve and to examine the residual of each model.

\section{ACKNOWLEDGEMENTS}

The authors wish to thank Dr. F.G. O'Neill, FRS Marine Laboratory Aberdeen for providing constructive comments and correcting the English text.

\section{REFERENCES}

1. Hamley JM. Review of gillnet selectivity. J. Fish. Res. Bd. Can. 1975; 32: 1943-1969.

2. Helser TE, Condrey RE, Geaghan JP. A new method of estimating gillnet selectivity, with an example for spotted seatrout, Cynocion nebulosus. Can. J. Fish. Aquat. Sci. 1991; 48: 487-492. 
3. Hansen MJ, Madenjian CP, Selgeby JH, Helser TE. Gillnet selectivity for lake trout (Salvelinus namaycush) in lake superior. Can. J. Fish. Aquat. Sci.1997; 54: 2483-2490.

4. Baranov FI. The capture of fish by gillnets. Mater. Poznoniyu Russ. Rybolov. 1914; 3: 56-99. (Partially transl. From Russian by W.E. Ricker)

5. Holt SJ. A method of determining gear selectivity and its application. ICNAF-ICES-FAO Joint Set. Meeting Paper 1957; S15: pp.21.

6. Ishida T. On the gill-net mesh selectivity curve. Bull. Hokkaido Reg. Fish. Res. Lab. 1962; 25: 20-25.

7. Kitahara T. On sweeping trammel net (kogisasiami) fishery along coast of the San'in district-III. Mesh selectivity curve of sweeping trammel net for branquillos. Bull. Jpn. Soc. Sci. Fish. 1968; 34: 759-763.

8. Yamamoto S, Meguro T, Shimazaki K. On the selectivity of the gill Net for pacific saury Cololabis saira Brevoort. Bull. Fac. Fish. Hokkaido Univ. 1982; 33: 240-248.

9. Koike A, Matuda K. Catching efficiency of trammel net with different slackness and mesh sizes of inner net. Bull. Jpn. Soc. Sci. Fish.1988; 54: 221-227.

10.Losanes LP, Matuda K, Machii T, Koike A. Catching efficiency and selectivity of entangling nets. Fish. Res. 1992; 13: 9-23.

11.Fujimori Y, Matuda K, Losanes LP, Koike A. Water tank experiment on the catching efficiency and mesh selectivity of gillnets. Nippon Suisan Gakkaishi 1990; 56: 2019-2027. 
12.Fujimori Y, Tokai T, Hiyama S, Matuda K. Selectivity and gear efficiency of trammel nets for kuruma prawn (Penaeus japonicus). Fish. Res. 1996; 26: 113-124.

13.Dickson W. Cod gillnet simulation model. Fish. Res. 1989; 7: 127-148.

14.Fujimori Y, Tokai T, Liang Z, Matuda K. Simulation model of capturing process of gillnets. Nippon Suisan Gakkaishi 1995; 61: 868-873.

15.Larkins HA. Comparison of salmon catches in monofilament and multifilament gill nets - Part II. Commer. Fish. Rev. 1964; 26: 1-7.

16.Pope JA, Margetts AR, Hamley JM, Akyuz EF. Manual of methods for fish stock assessment. Part III. Selectivity of fishing gear. FAO Fish. Tech. Pap. 1975; 41: 65 pp.

17.Hamley JM, Regier HA. Direct estimates of gillnet selectivity to walleye (Stizostedion vitreum vitreum). J. Fish. Res. Bd. Can. 1973; 30: 817-830.

18. Wulff A. Mathematical model for selectivity of gillnets. Arch. Fish. Wiss. 1986; 37: 101-106.

19.Millar RB. Estimating the size-selectivity of fishing gear by conditioning on the total catch. J. Am. Stat. Assoc. 1992; 87: 962-968.

20.Millar RB. The functional form of hook and gillnet selection curves cannot be determined from comparative catch data alone. Can. J. Fish. Aquat. Sci. 1994; 52: 883-891.

21.Xu X, Millar RB. Estimation of trap selectivity for male snow crab (Chionoecetes opilio) using the SELECT modeling approach with unequal sampling effort. Can. J. Fish. Aquat. Sci. 1993; 50: 2485-2490. 
22.Millar RB, Fryer RJ. Estimating the size-selection curves of towed gears, traps, nets and hooks. Rev. Fish Biol. Fish. 1999; 9; 89-116.

23. Nishiuchi S. Mesh selectivity of hair crab pots for male hair crabs (Erimacrus isenbeckii). Sci. Rep. Hokkaido Fish. Exp. Stn. 1999; 55: 131-139.

24.Tokai T, Kitahara T. Methods of determining the mesh selectivity curve of trawl net. Nippon Suisan Gakkaishi, 1989; 55: 643-649.

25.Regier HA, Robson DS. Selectivity of gill nets, especially to lake whitefish. J. Fish. Res. Bd. Can. 1966; 23: 423-454.

26.Losanes LP, Matuda K, Fujimori Y. Estimating the entangling effect of trammel and semi-trammel net selectivity on rainbow trout (Oncorhynchus mykiss). Fish. Res. 1992; 15: 229-242.

27.Madsen N, Holst R, Wileman D, Moth-Poulsen T. Size selectivity of sol gill nets fished in the north sea. Fish. Res. 1999; 44: 59-73.

28. Wileman DA, Ferro RST, Fonteyne R, Millar RB. Manual of methods of measuring the selectivity of towed fishing gears. ICES Coop. Res. Rep. 1996; No. 125: 126pp.

29.Dobson AJ. An introduction to generalized linear models. (Translation to Japanese by Tanaka Y, Morikawa T, Kurihara K) Kyoritsu Shuppan, Tokyo, 1993; 176pp.

30.Tokai T, Mitsuhashi T. Select model for estimating selectivity curve from comparative fishing experiment. Bull. Jpn. Soc. Fish. Oceanogr. 1998; 62: 235-247.

31.Fujimori Y, Tokai T. Estimation of gillnet selectivity curve by Ishida's 
method and Kitahara's method with MS-Excel. Bull. Jpn. Soc. Fish. Oceanogr. 1999; 63: 14-25.

32. Yamamoto S, Mishima S. Study on the suitable mesh size for salmon gill-net with results of measurement of netted fish. Bull. Fac. Fish. Hokkaido Univ. 1962; 13: 82-97.

33.Hovgård H. A two-step approach to estimating selectivity and fishing power of research gill nets used in Greenland waters. Can. J. Fish.Aquat. Sci. 1996; 53: 1007-1013. 


\section{Figure caption}

Fig. 1. Estimated master curves of selectivity for pink salmon.

Fig. 2(a). Observed (opened circle) and estimated (closed circle) catch proportion from the normal and the lognormal model.

Fig. 2(b). Observed (opened circle) and estimated (closed circle) catch proportion from the skew-normal and the bimodal normal model.

Fig. 3. Deviance residuals resulting from the fitting with each model. Open and closed circle shows the negative and positive residuals respectively, and the area of circle is proportional to the residual.

Fig. 4. Comparison between the relative fishing intensity from each model and the relative catch effort.

Fig. 5. Estimated selectivity curves and the selectivity plots calculated by Ishida's method for the $12.1 \mathrm{~cm}$ mesh size. 